Thermal Science and Engineering (2019) Volume 2 doi:10.24294/tse.v2i1.597

\title{
Thermal Processes in Thin Wires by Interaction with Microwave Radiation
}

\author{
N.G.Kokodii ${ }^{1,2}$, A.V.Shaposhnikova ${ }^{1}$, S.V.Pogorelov ${ }^{2}$ \\ ${ }^{1}$ Karazin Kharkov National University, 4 Svobody sq., Kharkov, Ukraine, 61022, E-mail: kokodiyng@ukr.net \\ ${ }^{2}$ National University of Pharmacy, 53 Pushkinskaya str., Kharkov, Ukraine, 61002, E-mail: svpog@yahoo.co.uk
}

\begin{abstract}
The effect of an anomalously large absorption of electromagnetic radiation in very thin conducting fibers was investigated. Efficiency factor of absorption can reach several hundred for a fiber with a diameter of several micrometers in the centimeter wavelength range. The effect can be used to transfer the energy of electromagnetic radiation to thin fibers without focusing radiation.

Keywords: Efficiency Factor of Absorption; Thin Fibers; Microwave Radiation; Heating.
\end{abstract}

\section{Introduction}

It is necessary to transfer the energy of laser or microwave radiation by small objects when solving many tasks of physics and technology: to spherical target in devices of laser thermonuclear synthesis, to active elements in fibre lasers and others. For effective energy transfer the radiation is focusing into a spot which dimensions should be comparable with the sizes of the target.

It was shown in works ${ }^{[1-4]}$ that electromagnetic radiation in the microwave range can be strongly absorbed in metallic wires or other thin conducting fibers, whose diameter is much smaller than the wavelength. Focusing radiation is not necessary. The dimensions of the focal spot can be thousands of times larger than the dimensions of the target. Efficiency factor of absorption (the ratio of the area of the effective target cross-section to the area of the geometric cross-section) can reach several thousand. It is necessary that the electric vector of the wave $\mathrm{E}$ be parallel to the axis of the wire.

This effect is very strongly in the microwave range with a wire diameter of several micrometers. In the experiment $^{[1]}$ a platinum wire with a diameter of $20 \mu \mathrm{m}$ placed in the radiation beam $3 \mathrm{~cm}$ in diameter at the wavelength of $1 \mathrm{~cm}$ about $10 \%$ of the beam energy was absorbed. The diameter of the beam was 1500 times larger than the diameter of the wire. Radiation beam on the wire was perpendicular to its axis.

Efficiency factor of absorption depends on the ratio between fiber diameter $D$ and radiation wavelength $\lambda$. At some values of $D / \lambda$ there is a maximum of absorption. In Figure 1 shown the dependence of the efficiency factor of absorption for graphite fiber on its diameter. When the wavelength increases the maximum shifts toward larger diameters and increases. At $\lambda=8$ $\mathrm{mm}$ the graphite fiber efficiency factor of absorption at the maximum reaches 78 for a fiber diameter of $5 \mu \mathrm{m}$; at $\lambda=10 \mathrm{~cm}$ it is 260 for a fiber diameter of $15 \mu \mathrm{m}$ and at $\lambda=1 \mathrm{~m}$ it is 780 for a fiber diameter of $50 \mu \mathrm{m}$.

In the case of the $\mathrm{H}$-wave (the magnetic vector of the wave is parallel to the fiber axis), efficiency factor of absorption is very small. Its value does not exceed 0.01 .

It was found in the work $^{[5]}$ that the energy absorption increases with an oblique incidence of the wave on the fiber. In Figure 2 shown the dependence of absorption of radiation with a wavelength of $1 \mathrm{~cm}$ in a graphite fiber with a diameter of $12 \mu \mathrm{m}$. The diffraction formulas of an electromagnetic wave on a circular cylinder were used by the calculations ${ }^{[6,7]}$.

Especially strong absorption is at angles of incidence greater than $60^{\circ} \mathrm{C}$. Efficiency factor of absorption E-wave there is several times larger than under normal incidence. Efficiency factor of absorption of $H$-wave is very small.

\footnotetext{
Copyright (C) 2019 N.G.Kokodii et al.

doi: 10.24294/tse.v2i1.597

EnPress Publisher LLC.This work is licensed under the Creative Commons Attribution-NonCommercial 4.0 International License (CC BY-NC 4.0).

http://creativecommons.org/licenses/ by/4.0/
} 


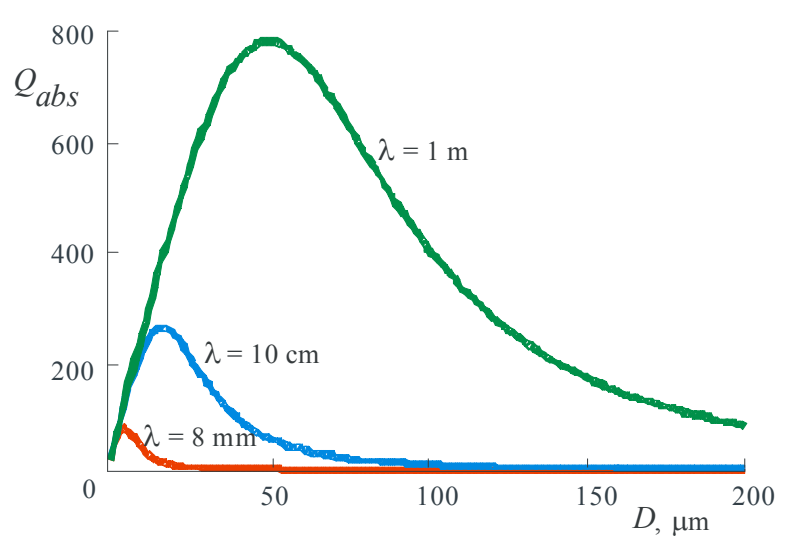

Figure 1; Efficiency factor of absorption for graphite fiber.

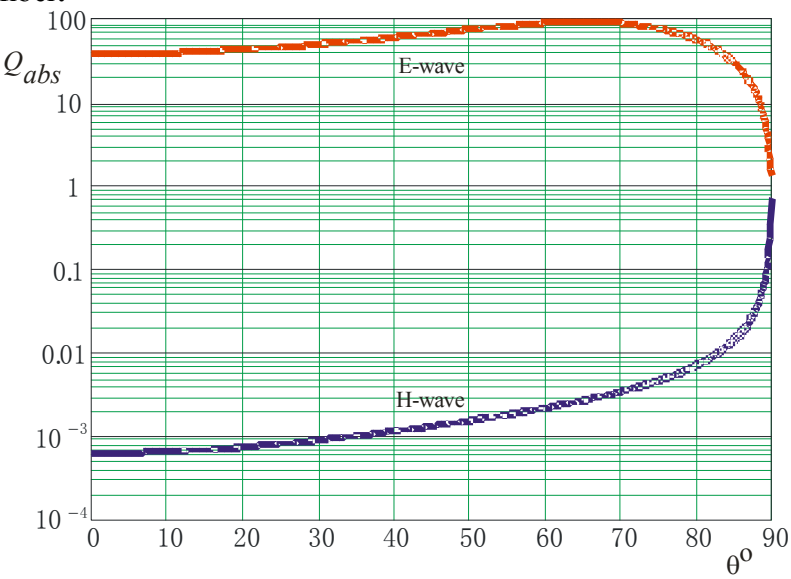

Figure 2; Dependence of the absorption efficiency factor on the angle of the wave incidence.

The nature of strong absorption at gliding fall of the wave is unclear. Absorption of energy in a conductor is always associated with the appearance of an electric current and hence with the presence of the longitudinal component of the electric field. But in the wave incident along the fiber the electric field vector is almost normal in the fiber axis. Probably the reason is the distortion of the waveform near the conductor.

\section{Experiment and results of measurements}

The absorption of microwave radiation in a thin graphite fiber at oblique incidence of the wave was measured in our experiment. The fiber diameter was $\mathrm{D}=$ $12 \mu \mathrm{m}$, the fiber length was $\mathrm{L}=280 \mathrm{~mm}$. The wavelength of the radiation was $1 \mathrm{~cm}$. The radiation power in the experiment was $0.5 \mathrm{~W}$. A thermistor wattmeter was used to monitor the power. The radiation beam was directed on the middle of the fiber at the angle $\theta=68^{\circ}$ to the normal. The beam profile is close to Gaussian. The root-mean-square radii of the beam cross-section at the point of incidence onto the

fiber were $r_{z}=4 \mathrm{~mm}$ in the longitudinal direction (along the fiber axis) and $r_{x}=7 \mathrm{~mm}$ in the transverse direction.

The absorption of radiation in the fiber causes it to heat up and change the electrical resistance. The multimeter M3500 was used to measure the resistance.

The resistance of the fiber was $R_{1}=31230 \mathrm{Ohm}$ at the temperature of $\mathrm{T}_{1}=20^{\circ} \mathrm{C}$. Its resistance decreased to $\mathrm{R}_{2}=31135 \mathrm{Ohm}$ under the action of radiation.

\subsection{Mathematical modeling of fiber heating by radiation}

The Gaussian beam of radiation falls on the fiber the profile of which is described by formula

$$
I(x, z)=I_{0} \exp \left[-\left(\frac{x^{2}}{r_{x}^{2}}+\frac{z^{2}}{r_{z}^{2}}\right)\right],
$$

where $I_{0}$ is the intensity on the beam axis.

The radiation intensity $I_{0}$ for a Gaussian beam is found by formula

$$
\begin{gathered}
I_{0}=\frac{P_{0}}{\pi r_{x} r_{z}} . \\
I_{0}=5684 \mathrm{~W} / \mathrm{m}^{2} \text { at } P_{0}=0.5 \mathrm{~W}, r_{z}=4 \mathrm{~mm} \text { and } r_{x}=7
\end{gathered}
$$
$\mathrm{mm}$.

Along the fiber axis the radiation intensity varies according to the law

$$
I(z)=I_{0} \exp \left(-\frac{z^{2}}{r_{z}^{2}}\right) .
$$

On the length $d z$ with coordinate $z$ the absorbed power is

$$
d P_{a b s}(z)=I(z) Q_{a b s} D d z,
$$

where $Q_{a b s}$ - efficiency factor of absorption. For the graphite fiber with the diameter of $12 \mu \mathrm{m}$, the radiation wavelength of $1 \mathrm{~cm}$ and an incidence angle of wave is $68^{\circ}$

$$
Q_{a b s}=65 .
$$

The power absorbed in the fiber is

$$
P_{a b s}=Q_{a b s} D \int_{-\frac{L}{2}}^{\frac{L}{2}} I(z) d z=0.031 \mathrm{~W}
$$

Consequently about $6 \%$ of the incident power is absorbed in the fiber despite the fact that the diameter of the beam is 500 times larger than the diameter of the fiber.

We make three assumptions for the estimate the heating temperature of the fiber:

1. The temperature over the entire cross section of the 
fiber is the same.

2. The temperature distribution along the fiber repeats the distribution of the intensity of the incident radiation.

3. Thermo-physical parameters of the fiber (heat capacity, thermal conductivity, heat exchange with the external medium, etc.) do not depend on temperature.

Paragraphs 1 and 2 are associated with a small fiber thickness and a small heat flux along it. The solution of the thermal task (here it is not shown because of the large volume) confirms it.

Paragraph 3 at strong heating (several hundred degrees) is not true. But taking these dependences into account when solving the thermal task shows that the efficiency of the transmission of radiation energy to the fiber is greater than the estimate made at assumption 3 . Therefore this estimate is the minimum possible.

The Newton law for the heat exchange of a heated object with an external medium shows that an increase in body temperature with a heating power $\mathrm{P}_{\mathrm{abs}}$ is

$$
T=\frac{P_{a b s}}{\alpha_{P} L} \text {. }
$$

Here $\alpha_{P}$ is the linear coefficient of heat exchange with the external medium. It shows the power flowing into the external medium from a fiber $1 \mathrm{~m}$ long at a temperature difference of fiber and medium of $1 \mathrm{~K}$. For a thin fiber it does not depend on the diameter ${ }^{[8]}$.

Using equations (1) - (3) we obtain an expression for the temperature distribution along the fiber:

$$
T(z)=\frac{I_{0} Q_{a b s} D}{\alpha_{P}} \exp \left(-\frac{z^{2}}{r_{z}^{2}}\right) .
$$

With the values of the radiation beam and fiber parameters listed above and the parameter $\alpha_{P}=0.02$ $\mathrm{W} /(\mathrm{m} \mathrm{K})$ measured experimentally the temperature at the maximum is $242^{\circ} \mathrm{C}$. Figure 3 shows the temperature distribution along the fiber obtained with the thermal imager. It is shown that the size of the fiber section heated to a high temperature is approximately $8 \mathrm{~mm}$, i.e. to the beam diameter along the $\mathrm{z}$ axis. The temperature scale in the lower part of the figure shows that the temperature of the heated part of the fiber is more than $200^{\circ} \mathrm{C}$ (red color). The temperature of the other sections of the fiber (yellow color) is several tens of degrees due to the heating of the fiber by the current of the resistance meter. This is consistent with the results of calculations.

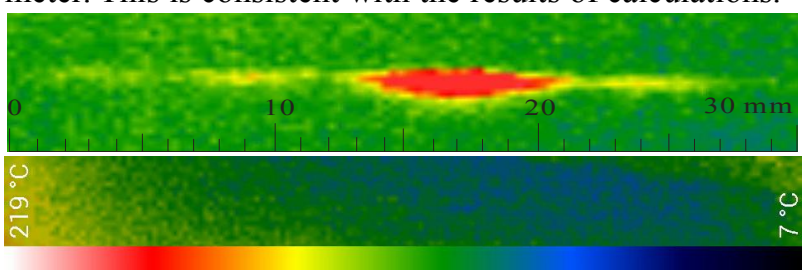

Figure 3; Thermal picture of fiber heating by radiation.

In Figure 4 shown the result of digital processing of the picture in Figure 3. The reason for the unevenness of the graph is the graininess of the picture. The solid line is a smoothed graph of temperature distribution along the fiber. Qualitatively it agrees with Figure 3 and the results of calculations.

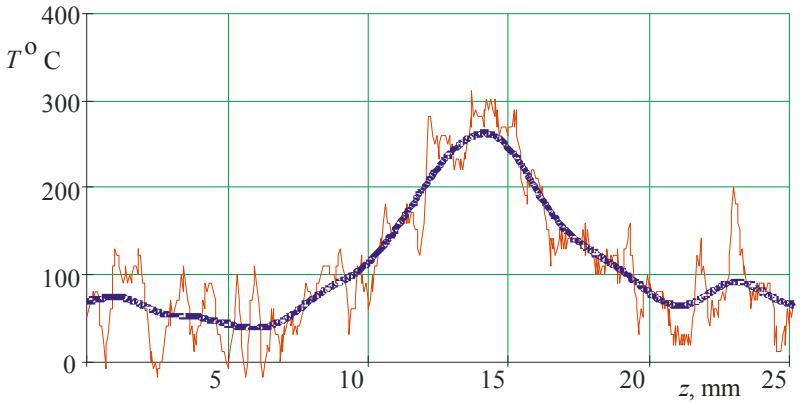

Figure 4; Temperature distribution along the fiber.

Dependence of the resistance to the temperature is given by:

$$
R(T)=R_{0}\left(1+\alpha_{R} T\right)
$$

where $\mathrm{R}_{0}$ is the resistance at $0^{\circ} \mathrm{C}, \alpha_{R}=-0.000341 / \mathrm{K}$ is the temperature coefficient of the resistance of graphite. It was determined experimentally for used fiber since it depends strongly on the method of its manufacture. It is negative. This means that the resistance of the fiber decreases with heating.

According to this formula it was found that $\mathrm{R}_{0}=$ $31440 \mathrm{Ohm}$.

The conductivity at $0^{\circ} \mathrm{C}$ was found from the equation which is connecting the conductivity of the fiber and its resistance:

$$
\sigma_{0}=\frac{4 L}{\pi D^{2} R_{0}}=7.87 \cdot 10^{4} \frac{1}{\Omega \cdot m}
$$

Dependence of the conductivity to the temperature is as follows:

$$
\sigma=\frac{1}{1+\alpha_{R} T}
$$

The resistance of a fiber length $\mathrm{dz}$ is: 


$$
d R(z)=\frac{4 d z}{\pi D^{2} \sigma} .
$$

Then the resistance of the whole fiber:

$$
R=\frac{4}{\pi D^{2} \sigma_{0}} \int_{-\frac{L}{2}}^{\frac{L}{2}}\left[1+\alpha_{R} T(z)\right] d z
$$

Calculations by this formula using expression (4) for the temperature distribution along the fiber give such a resistance value of the "hot" fiber:

$$
\mathrm{R}=31125 \text { Ohm. }
$$

In the experiment was obtained $-R=31135 \mathrm{Ohm}$. This is in good consistent. Therefore we can assume that the mathematical model correctly describes the process of fiber heating by electromagnetic radiation and the estimate of the absorbed radiation power in the fiber is $0.031 \mathrm{~W}$ and the absorption coefficient is $6 \%$ and corresponds to the truth.

\subsection{Processing of measurement results - absorbed power in the fiber}

We can find the heating temperature of the fiber by radiation by using equation (5):

$$
T_{2}=\frac{1}{\alpha_{R}}\left[\frac{R_{2}}{R_{1}}\left(1+\alpha_{R} T_{1}\right)-1\right] .
$$

Calculations by this formula give $T_{2}=28.9^{\circ} \mathrm{C}$. This is the average temperature over the length of the fiber. It was shown above that in some places it exceeds $200^{\circ} \mathrm{C}$.

Absorbed in the fiber power can be calculated from the formula

$$
P_{a b s}=\alpha_{P} L\left(T_{2}-T_{1}\right),
$$

which follows from equation (3). Substituting the values obtained in the experiment here we get:

$$
P_{a b s}=0.05 \mathrm{~W}
$$

Consequently $10 \%$ of the energy of the incident radiation is absorbed in the fiber. The difference between this value and the value obtained as a result of mathematical modeling is explained by the assumptions made in the theoretical estimate of the absorbed power and the inaccuracy in calculating the efficiency factor of absorption by the formulas of the diffraction theory. But both theory and experiment confirm the existence effect of an anomalously large absorption of electromagnetic radiation in very thin conducting fibers and the possibility of using it in engineering.

\section{Conclusion}

There is an effect of strong absorption of microwave radiation in very thin conducting fibers. This effect is enhanced by the sliding fall of the wave on the fiber. Experimental confirmation of this effect was given. In the graphite fiber with a diameter of $12 \mu \mathrm{m}$ was absorbed $10 \%$ of the energy of the microwave radiation, which was incident to it at an angle of $68^{\circ}$ with a wavelength of $1 \mathrm{~cm}$.

The diameter of the radiation beam was about 6 $\mathrm{mm}$ which is 500 times larger than the fiber diameter. There is no need to focus the beam.

\section{Reference}

1. V.M. Kuz'michev, N.G. Kokodiy, B.V. Safronov, V.P. Balkasin. Values of the absorption efficiency factor of a thin metal cylinder in the microwave band. Journal of Communication Technology and Electronics, 2003, Vol. 48, No. 11, pp. 1240-1242.

2. N.G. Kokodiy. Microwave radiation absorption in an ultrathin double-layer cylinder. Journal of Communication Technology and Electronics, 2006, Vol. 51, No. 2, pp. 175-178.

3. Shi He, S.N. Shulga, N.G. Kokodity et al. Interaction of electromagnetic waves in a waveguide with very thin wires. Journal of Communication Technology and Electronics, 2011, Vol. 56, No. 10, pp. 1193-1196.

4. A. Akhmeteli, N.G. Kokodiy, B.V. Safronov, V.P. Balkashin, I.A. Priz, A. Tarasevitch. Efficient non-resonant absorption in thin cylindrical targets: experimental evidence for longitudinal geometry. ArXiv: 1208.0066v3 [physics:plasm-ph] 6 Aug 2013. - pp. 1-15

5. N.G. Kokodii, M.V. Kaydash, V.A. Timaniuk. Interaction of electromagnetic radiation with a thin metal wire in the case of a glancing incident wave. Journal of Communication Technology and Electronics, 2017, Vol. 62, No. 3, pp. 205-211.

6. J.R. Wait. Scattering of a plane wave from a circular dielectric cylinder an oblique incidence. Canadian Journal of Physics, 1955, Vol. 33, No. 5, pp. 189-195.

7. A.C. Lind, J.M. Greenberg. Electromagnetic scattering by obliquely oriented cylinders. Journal of Applied Physics, 1966, Vol. 37, No. 8, pp. 3195-3203.

8. R.C.L. Bosworth. Heat transfer phenomena. The flow of heat in physical systems. - John Wiley and Sons. N.Y., 1952 